Portland State University

PDXScholar

$1-1-2010$

\title{
On Residual Lifetimes of k-out-of-n Systems With Nonidentical Components
}

\author{
Subhash C. Kochar \\ Portland State University, kochar@pdx.edu \\ Maochao Xu \\ Portland State University
}

Follow this and additional works at: https://pdxscholar.library.pdx.edu/mth_fac

Part of the Mathematics Commons

Let us know how access to this document benefits you.

Citation Details

Kochar, S., \& Xu, M. (2010). On residual lifetimes of k-out-of-n systems with nonidentical components. Probability in the Engineering \& Informational Sciences, 24(1), 109-127.

This Article is brought to you for free and open access. It has been accepted for inclusion in Mathematics and Statistics Faculty Publications and Presentations by an authorized administrator of PDXScholar. Please contact us if we can make this document more accessible: pdxscholar@pdx.edu. 


\section{Probability in the Engineering and Informational Sciences}

http://journals.cambridge.org/PES

Additional services for Probability in the Engineering and Informational

Sciences:

Email alerts: Click here

Subscriptions: Click here

Commercial reprints: Click here

Terms of use : $\underline{\text { Click here }}$

\section{ON RESIDUAL LIFETIMES OF $k$-OUT-OF- $\boldsymbol{n}$ SYSTEMS WITH NONIDENTICAL COMPONENTS}

Subhash Kochar and Maochao Xu

Probability in the Engineering and Informational Sciences / Volume 24 / Issue 01 / January 2010, pp 109 - 127

DOI: 10.1017/S0269964809990167, Published online: 21 December 2009

Link to this article: http://journals.cambridge.org/abstract S0269964809990167

How to cite this article:

Subhash Kochar and Maochao Xu (2010). ON RESIDUAL LIFETIMES OF $k$-OUT-OF- $n$ SYSTEMS WITH NONIDENTICAL COMPONENTS. Probability in the Engineering and Informational Sciences, 24, pp 109-127 doi:10.1017/S0269964809990167

Request Permissions : $\underline{\text { Click here }}$ 


\title{
ON RESIDUAL LIFETIMES OF $k$-OUT-OF- $n$ SYSTEMS WITH NONIDENTICAL COMPONENTS
}

\author{
Subhash Kochar and Maochao Xu \\ Department of Mathematics and Statistics \\ Portland State University \\ Portland, OR 97201 \\ E-mail:maochao@pdx.edu
}

\begin{abstract}
In this article, mixture representations of survival functions of residual lifetimes of $k$-out-of- $n$ systems are obtained when the components are independent but not necessarily identically distributed. Then we stochastically compare the residual lifetimes of $k$-out-of- $n$ systems in one- and two-sample problems. In particular, the results extend some results in Li and Zhao [14], Khaledi and Shaked [13], Sadegh [17], Gurler and Bairamov [7] and Navarro, Balakrishnan, and Samaniego [16]. Applications in the proportional hazard rates model are presented as well.
\end{abstract}

\section{INTRODUCTION}

Let $X_{1}, X_{2}, \ldots, X_{n}$ be independent nonnegative continuous random variables. In the reliability theory context, order statistics $X_{1: n} \leq X_{2: n} \leq \cdots \leq X_{n: n}$ are used to denote lifetimes of systems. For example, $X_{n-k+1: n}$ represents the lifetime of a $k$-out-of- $n$ system. In particular, the parallel and series systems are 1-out-of- $n$ and $n$-out-of- $n$ systems. The residual life of a unit with lifetime $X$ at time $t$ is denoted by

$$
(X)_{t}=[X-t \mid X>t]
$$

and it describes the residual time of the unit given that it is working at time $t>0$. Recently, the residual life of a $k$-out-of- $n$ system given the information that at most $r$ components have failed but the system is still working has received great attention. Bairamov, Ahsanullah, and Akhundov [3] investigated the mean residual life of a parallel system under the condition that none of the components of the system has 
failed at time $t$; that is,

$$
\left[X_{n: n}-t \mid X_{1: n}>t\right]
$$

Subsequently, Asadi and Bairamov [1] studied the mean residual life of a parallel system under the condition that at least $(n-k+1)$ components of the system are working at time $t$ :

$$
\left[X_{n: n}-t \mid X_{k: n}>t\right]
$$

Asadi and Bairamov [2] investigated the mean residual life of an $(n-k+1)$-out-of$n$ system under the condition that at time $t$, all of the components of the system are working: $\left[X_{k: n}-t \mid X_{1: n}>t\right]$. Recently, Li and Zhao [14] considered this problem in more generality. They considered the residual life of an $(n-k+1)$-out-of- $n$ system given the condition that at least $(n-i+1)$ components of the system are working: for $1 \leq i \leq k \leq n$,

$$
\left[X_{k: n}-t \mid X_{i: n}>t\right]
$$

$\mathrm{Hu}$, Jin, and Khaledi [8] studied the conditional distributions of generalized order statistics, which include the residual lifetimes of $k$-out-of- $n$ systems as a special case. Khaledi and Shaked [13] considered the residual life of a coherent system given at least $(n-i+1)$ components of the system are working and gave the motivation for this research. Consider a situation where a system is equipped with an alarm system that gets activated when a certain number of components in the system fail. It is of interest for the engineer to know the behavior of the residual life of the system for the maintenance purpose. This problem has also potential applications in medical research. For example, a doctor might like to know the residual life of a patient after one of his/her kidneys has failed.

Most of the results obtained in the literatures are restricted to the case when the components are independent and identically (i.i.d.) distributed. In many practical situations, however, systems might be composed of independent but nonidentical components. To the best of our knowledge, this general case has been considered by Sadegh [17] for the first time. He considered the mean residual life of a parallel system with independent but nonidentical components, which successfully extends some results in Asadi and Bairamov [1]. Recently, Gurler and Bairamov [7] investigated the mean residual life functions of parallel systems and $k$-out- $n$ systems under the condition that all components are alive at time $t$. Zhao, Li, and Balakrishnan [19] considered the residual life of an $(n-k+1)$-out-of- $n$ system given that the $l$ th component has failed but the $(l+1)$ st component is working at time $t \geq 0$; that is,

$$
\left[X_{k: n}-t \mid X_{l: n} \leq t<X_{l+1: n}\right],
$$

where $1 \leq l<k \leq n$. In this article, we pursue this topic further and study the residual lifetime of an $(n-k+1)$-out-of- $n$ system with independent but nonidentical 
components under very general conditions. More precisely, we will study the residual lifetime of an $(n-k+1)$-out-of- $n$ system given the condition that at least $(n-l+1)$ components of the system are working at time $t$ :

$$
X_{k, l, n, t}=\left[X_{k: n}-t \mid X_{l: n}>t\right] \quad \text { for } 1 \leq l \leq k \leq n .
$$

We also consider the other realistic and interesting situation: the residual lifetime of an $(n-k+1)$-out-of- $n$ system given the condition that at least $l$ components of the system have failed but the system is working at time $t$ :

$$
\tilde{X}_{k, l, n, t}=\left[X_{k: n}-t \mid X_{l: n} \leq t, X_{k: n}>t\right] \quad \text { for } 1 \leq l<k \leq n
$$

This situation often occurs in practice. For example, a four-engine plane in which two engines must work for the plane to fly is equipped with a device that alerts the failure time of engines. It is extremely important for the engineer to know the properties of the residual life of the four-engine system in order to make a good system's design. It is interesting to note that Navarro, Balakrishnan, and Samaniego [16] considered the residual lifetime of a coherent system $T$ with i.i.d. components under the same scenario (i.e., $\left[T-t \mid X_{l: n} \leq t, T>t\right]$ ), where they obtained a mixture representation for the survival function of the system's residual life. They also posed one interesting question of whether one could obtain useful representation results in cases when systems have components that are independent but not necessarily identically distributed. In this article, we will answer the question by providing a mixture expression for the survival function of $\tilde{X}_{k, l, n, t}$, the residual lifetime of an $(n-k+1)$-out-of- $n$ system given the condition that at least $l$ components of the system have failed but the system is working at time $t$.

We will also stochastically compare the residual lifetimes of $(n-k+1)$-out-of$n$ systems in one-sample and two-sample problems with nonidentical components. This work extends the corresponding results in Li and Zhao [14], Khaledi and Shaked [13], Sadegh [17], and Gurler and Bairamov [7].

The article is organized as follows. In Section 2 we review some concepts. The mixture representations of survival functions of residual lifetimes of $(n-k+1)$ out-of- $n$ systems are given in Section 3. The mean residual lifetime functions of $(n-k+1)$-out-of- $n$ systems are also obtained there. In Section 4 we will stochastically compare the residual lifetimes of $(n-k+1)$-out-of- $n$ systems with nonidentical components from one sample and two samples. In the last section, some examples and applications are given for the proportional hazard rates model.

\section{PRELIMINARIES}

The joint distribution functions of order statistics can be conveniently represented as permanents when the underlying random variables are not identical. One can refer to Bapat and Kochar [5], Hu, Zhu, and Wei [11], Hu, Wang, and Zhu [10], and Balakrishnan [4] for related topics. 
If $\mathbf{A}=\left(a_{i, j}\right)$ is an $n \times n$ matrix, then the permanent of $\mathbf{A}$ is defined as

$$
\operatorname{Per} \mathbf{A}=\sum_{\sigma} \prod_{i=1}^{n} a_{i, \sigma(i)},
$$

where the summation is taken over all permutations $\sigma=(\sigma(1), \ldots, \sigma(n))$ of $(1, \ldots, n)$. If $\mathbf{a}_{1}, \mathbf{a}_{2}, \ldots$, are column vectors, then the permanent

$$
\operatorname{Per} \mathbf{A}=[\underbrace{\mathbf{a}_{1}}_{r_{1}}, \underbrace{\mathbf{a}_{2}}_{r_{2}}, \cdots,]
$$

is obtained by taking $r_{1}$ copies of $\mathbf{a}_{1}, r_{2}$ copies of $\mathbf{a}_{2}$, and so on, and

$$
[\underbrace{\mathbf{a}_{1}}_{r_{1}}, \underbrace{\mathbf{a}_{2}}_{r_{2}}, \cdots,]_{C}
$$

means that the permanent has those rows only in $C$.

Similar to the determinant, one could use Laplace expansion for the permutation along any row or column. For example, if $\mathbf{A}(i, j)$ denotes the matrix $\mathbf{A}$ by deleting row $i$ and column $j$, then

$$
\begin{aligned}
& \operatorname{Per} \mathbf{A}=\sum_{j=1}^{n} a_{i j} \operatorname{Per} \mathbf{A}(i, j) \quad \text { for } i=1, \ldots, n, \\
& \text { Per } \mathbf{A}=\sum_{i=1}^{n} a_{i j} \operatorname{Per} \mathbf{A}(i, j) \quad \text { for } j=1, \ldots, n .
\end{aligned}
$$

For mutually independent random variables $X_{1}, \ldots, X_{n}$, let $F_{i}$ and $\bar{F}_{i}$ be the distribution and survival functions of $X_{i}, i=1, \ldots, n$, respectively. The column vector $\left(F_{1}(x), \ldots, F_{n}(x)\right)^{\prime}$ will be denoted by $\mathbf{F}(x) . \overline{\mathbf{F}}(x)$ is similarly defined for the vector of survival functions. The distribution and survival functions of the residual life of $X_{i}$ at time $t$ are denoted by $F_{i, t}(x)$ and $\bar{F}_{i, t}(x)$, respectively. The column vector $\left(F_{1, t}(x), \ldots, F_{n, t}(x)\right)^{\prime}$ will be denoted simply by $\mathbf{F}_{\mathbf{t}}(x)$, and $\overline{\mathbf{F}}_{\mathbf{t}}(x)$ is defined similarly. Note that the survival function of an $(n-r+1)$-out-of- $n$ system has the following permanent expression (cf. Balakrishnan, [4]). For $1 \leq r \leq n, x \geq 0$,

$$
\begin{aligned}
\bar{F}_{r, n}(x) & =P\left(X_{r: n}>x\right) \\
& =\sum_{i=0}^{r-1} P(\text { exactly } i \text { of } X \text { 's are } \leq x) \\
& =\sum_{i=0}^{r-1} \frac{1}{i !(n-i) !}[\underbrace{\mathbf{F}(\mathbf{x})}_{i}, \underbrace{\overline{\mathbf{F}}(\mathbf{x})}_{n-i}] .
\end{aligned}
$$


Now, let us review some stochastic orders that will be used in the sequel. Let $X$ and $Y$ be two nonnegative random variables with survival functions $\bar{F}$ and $\bar{G}$, respectively.

DEFINITION 2.1 (Shaked and Shanthikumar, [18]): $X$ is said to be smaller than $Y$ in the following:

(i) hazard rate order (denoted by $X \leq_{\mathrm{hr}} Y$ ) if $\bar{G}(x) / \bar{F}(x)$ is increasing in $x$;

(ii) stochastic order (denoted by $X \leq$ st $Y$ ) if $\bar{F}(x) \leq \bar{G}(x)$ for all $x$.

It is known that

$$
X \leq_{\mathrm{hr}} Y \Rightarrow X \leq_{\mathrm{st}} Y
$$

and, for $t \geq 0$,

$$
X \leq_{\mathrm{hr}} Y \Leftrightarrow(X)_{t} \leq_{\mathrm{st}}(Y)_{t}
$$

\section{MIXTURE REPRESENTATIONS OF RESIDUAL LIFE FUNCTIONS}

\subsection{Survival Function of $X_{k, l, n, t}$}

The survival function of the residual life of an $(n-k+1)$-out-of- $n$ system given at least $(n-l+1)$ components of the system are working at time $t \geq 0$, for $1 \leq l \leq$ $k \leq n$

$$
X_{k, l, n, t}=\left[X_{k: n}-t \mid X_{l: n}>t\right],
$$

could be represented as the following mixture form.

THEOREM 3.1: For $1 \leq l \leq k \leq n$ and $t, x \geq 0$,

$$
\begin{aligned}
\bar{F}_{k, l, n, t}(x) & =P\left(X_{k, l, n, t}>x\right) \\
& =\frac{\sum_{i=0}^{l-1} \sum_{C_{i}} \phi_{i}(t) \bar{F}_{k-i, n-i, t}^{\left(C_{i}^{c}\right)}(x)}{\sum_{i=0}^{l-1} \sum_{C_{i}} \phi_{i}(t)},
\end{aligned}
$$

where the summation $C_{i}$ with cardinality $i$ extends over all subsets of $\{1,2, \ldots, n\}$ and $C_{0} \equiv \emptyset$ and $C_{i}^{c}$ is the corresponding complement. Here,

$$
\phi_{i}(t)=\prod_{s \in C_{i}} \frac{F_{s}(t)}{\bar{F}_{s}(t)} ;
$$

for $i=1, \ldots, l-1$ and $\phi_{0}(t)=1$,

$$
\bar{F}_{k-i, n-i, t}^{\left(C_{i}^{c}\right)}(x)=\sum_{j=0}^{k-i-1} \frac{1}{j !(n-i-j) !}[\underbrace{\mathbf{F}_{\mathbf{t}}(\mathbf{x})}_{j}, \underbrace{\overline{\mathbf{F}}_{t}(\boldsymbol{X})}_{n-i-j}]_{C_{i}^{c}},
$$

which denotes the survival function of $X_{k-i, n-i, t}$, where $X_{k-i, n-i, t}$ is the $(k-i)$ th-order statistic from $\left(X_{j}\right)_{t}, j \in C_{i}^{c}$. 
ProOF: Note that, for $1 \leq l \leq k \leq n, t, x \geq 0$,

$$
\begin{aligned}
& P\left(X_{k: n}>t+x, X_{l: n}>t\right) \\
& =\sum_{i=0}^{l-1} \sum_{j=0}^{k-i-1} P(\text { exactly } i \text { of } X \text { 's are } \leq t, t<\text { exactly } j \text { of } X \text { 's are } \leq t+x) \\
& =\sum_{i=0}^{l-1} \sum_{j=0}^{k-i-1} \frac{1}{i ! j !(n-i-j) !}[\underbrace{\mathbf{F}(\mathbf{t})}_{i}, \underbrace{\mathbf{F}(\mathbf{t}+\mathbf{x})-\mathbf{F}(\mathbf{t})}_{j}, \underbrace{\overline{\mathbf{F}}(\mathbf{t}+\mathbf{x})}_{n-i-j}] \\
& =\sum_{i=0}^{l-1} \sum_{j=0}^{k-i-1} \frac{1}{i ! j !(n-i-j) !} \sum_{C_{i}}[\underbrace{\mathbf{F}(\mathbf{t})}_{i}]_{C_{i}}[\underbrace{\mathbf{F}(\mathbf{t}+\mathbf{x})-\mathbf{F}(\mathbf{t})}_{j}, \underbrace{\overline{\mathbf{F}}(\mathbf{t}+\mathbf{x})}_{n-i-j}]_{C_{i}^{c}} \\
& =\prod_{i=1}^{n} \bar{F}_{i}(t) \sum_{i=0}^{l-1} \sum_{j=0}^{k-i-1} \sum_{C_{i}} \prod_{s \in C_{i}} \frac{F_{s}(t)}{\bar{F}_{s}(t)} \frac{1}{j !(n-i-j) !}[\underbrace{\mathbf{1}-\overline{\mathbf{F}}_{\mathbf{t}}(\mathbf{x})}_{j}, \underbrace{\overline{\mathbf{F}}_{\mathbf{t}}(\mathbf{x})}_{n-i-j}]_{C_{i}^{c}} \\
& =\prod_{i=1}^{n} \bar{F}_{i}(t) \sum_{i=0}^{l-1} \sum_{C_{i}} \prod_{s \in C_{i}} \frac{F_{S}(t)}{\bar{F}_{S}(t)} \sum_{j=0}^{k-i-1} \frac{1}{j !(n-i-j) !}[\underbrace{\mathbf{F}_{\mathbf{t}}(\mathbf{x})}_{j}, \underbrace{\overline{\mathbf{F}}_{\mathbf{t}}(\mathbf{x})}_{n-i-j}]_{C_{i}^{c}} \\
& =\prod_{i=1}^{n} \bar{F}_{i}(t) \sum_{i=0}^{l-1} \sum_{C_{i}} \phi_{i}(t) \bar{F}_{k-i, n-i, t}^{\left(C_{i}^{c}\right)}(x) .
\end{aligned}
$$

Observing that

$$
P\left(X_{l: n}>t\right)=\prod_{i=1}^{n} \bar{F}_{i}(t) \sum_{i=0}^{l-1} \sum_{C_{i}} \phi_{i}(t),
$$

the result follows immediately.

Remarks:

(a) It is seen that $\bar{F}_{k-i, n-i, t}^{\left(C_{i}^{c}\right)}(x)$ is actually the survival function of an $(n-k+1)$ out-of- $(n-i)$ system composed of $(n-i)$ used units with residual life $\left(X_{j}\right)_{t}$, $j \in C_{i}^{c}$. If $t=0$, it reduces to the form of Eq. (2.1).

(b) If $X_{i}$ 's are i.i.d. random variables,

$$
\bar{F}_{k, l, n, t}(x)=\frac{\sum_{i=0}^{l-1}\left(\begin{array}{c}
n \\
i
\end{array}\right) \phi_{i}(t) \bar{F}_{k-i, n-i, t}(x)}{\sum_{i=0}^{l-1}\left(\begin{array}{c}
n \\
i
\end{array}\right) \phi_{i}(t)},
$$

which reduces to the expression of $\bar{H}_{l, k, n, t}$ in Li and Zhao [14]. 
(c) For the particular case $l=1$, we have the following interesting result:

$$
\bar{F}_{k, 1, n, t}(x)=\sum_{j=0}^{k-1} \frac{1}{j !(n-j) !}[\underbrace{\mathbf{F}_{\mathbf{t}}(\mathbf{x})}_{j}, \underbrace{\overline{\mathbf{F}}_{\mathbf{t}}(\mathbf{x})}_{n-j}] ;
$$

that is,

$$
X_{k, 1, n, t} \stackrel{\text { st }}{=} X_{k, n, t},
$$

This result states that if all of the components are working at time $t$, the residual lifetime of an $(n-k+1)$-out- $n$ system is stochastically equal to that of an $(n-k+1)$-out- $n$ system composed of $n$ used components with residual life $\left(X_{i}\right)_{t}$. It follows from Theorem 1.B.26 of Shaked and Shanthikumar [18] that, for $k=1, \ldots, n-1$,

$$
X_{k, 1, n, t} \leq \mathrm{hr} X_{k+1,1, n, t} .
$$

By Theorem 1.B. 28 of Shaked and Shanthikumar [18], it holds that for $k=$ $1, \ldots, n-1$,

$$
X_{k, 1, n, t} \leq_{\mathrm{hr}} X_{k, 1, n-1, t},
$$

which extends Theorem 2 of Sadegh [17]. It also follows from Theorem 1.B.27 of Shaked and Shanthikumar [18] that if $X_{j} \leq_{\mathrm{hr}} X_{n}$, which implies $\left(X_{j}\right)_{t} \leq_{\mathrm{hr}}$ $\left(X_{n}\right)_{t}$, for all $j=1, \ldots, n-1$, then for $k=2, \ldots, n$,

$$
X_{k-1,1, n-1, t} \leq_{\mathrm{hr}} X_{k, 1, n, t} .
$$

Example 3.2: Suppose a data processing system has four video displays and that a minimum of two displays operable are required for full data display. Hence, this display subsystem is a 2-out-of-4 system. Now, suppose at time $t$, at most one display failed. We are interested in the residual lifetime of this system, which could be expressed as $X_{3,2,4, t}=\left[X_{3: 4}-t \mid X_{2: 4}>t\right]$. From Theorem 3.1,

$$
\bar{F}_{3,2,4, t}(x)=\frac{\bar{F}_{3,4, t}(x)+\sum_{C_{1}} \phi_{1}(t) \bar{F}_{2,3, t}^{\left(C_{1}^{c}\right)}(x)}{1+\sum_{C_{1}} \phi_{1}(t)} .
$$

For simplicity, let us assume that $X_{i}$ has an exponential distribution with survival function

$$
\bar{F}_{i}(x)=e^{-\lambda_{i} x} .
$$

Then

$$
\bar{F}_{3,4, t}(x)=e^{-\sum_{i=1}^{4} \lambda_{i} x}+\sum_{C_{3}, j \in C_{3}^{c}} e^{-\sum_{s \in C_{3}} \lambda_{s} x}\left(1-e^{-\lambda_{j} x}\right)+\sum_{C_{2}} e^{-\sum_{s \in C_{2}} \lambda_{s} x} \prod_{j \in C_{2}^{c}}\left(1-e^{-\lambda_{j} x}\right),
$$


where the summation $C_{i}$ with size $i$ extends over all subsets of $\{1,2,3,4\}$ and $C_{i}^{c}$ is the corresponding complement.

$$
\sum_{C_{1}} \phi_{1}(t) \bar{F}_{2,3, t}^{\left(C_{1}^{c}\right)}(x)=\sum_{i=1}^{4}\left[e^{\lambda_{i} t}-1\right]\left[e^{-\sum_{j \neq i} \lambda_{i} x}+\sum_{C_{2}^{(i)}, j \in\left(C_{2}^{(i)}\right)^{c}} e^{-\sum_{s \in C_{2}^{(i)} \lambda_{s} x}}\left(1-e^{-\lambda_{j} x}\right)\right],
$$

where the summation $C_{2}^{(i)}$ with size 2 extends over all subsets of $\{1,2,3,4\} /\{i\}$, and $\left(C_{2}^{(i)}\right)^{c}$ having size 1 means the corresponding complement,

$$
\sum_{C_{1}} \phi_{1}(t)=\sum_{i=1}^{4} e^{\lambda_{i} t}-4
$$

Now, assume

$$
\left(\lambda_{1}, \lambda_{2}, \lambda_{3}, \lambda_{4}\right)=(0.1,0.3,0.5,0.7)
$$

Figure 1 shows the survival function of the system's residual lifetime given that at most one component failed at time $t=10$.

Gurler and Bairamov [7] derived the mean residual life functions for $(n-k+$ 1)-out-of- $n$ systems given that all of the components are working at time $t$. From Theorem 3.1, one can easily derive the following general mixture expression for the mean residual life functions of $(n-k+1)$-out-of- $n$ systems.

Proposition 3.3: For $1 \leq l \leq k \leq n$ and $t, x \geq 0$,

$$
\mu_{k, l, n}(t)=\mathrm{E}\left(X_{k, l, n, t}\right)=\frac{\sum_{i=0}^{l-1} \sum_{C_{i}} \phi_{i}(t) \mu_{k, i, n}^{\left(C_{C}^{c}\right)}(t)}{\sum_{i=0}^{l-1} \sum_{C_{i}} \phi_{i}(t)},
$$

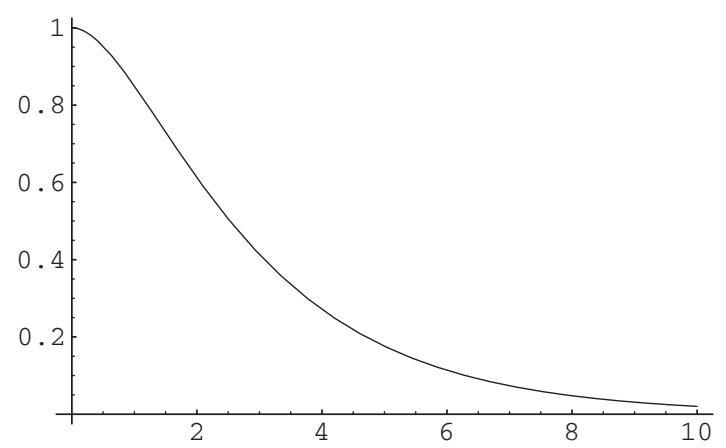

Figure 1. The survival function of the residual lifetime of the 2-out-of-4 system given that at most one component is failed at time $t=10$. 
where

$$
\mu_{k, i, n}^{\left(C_{i}^{c}\right)}(t)=\mathrm{E}\left(X_{k-i, n-i, t}\right)=\int_{0}^{\infty} \bar{F}_{k-i, n-i, t}^{\left(C_{i}^{c}\right)}(x) d x .
$$

The case $l=1$,

$$
\mu_{k, 1, n}(t)=\mathrm{E}\left(X_{k, n, t}\right)
$$

reduces to the expression given in Eq. (6) of Gurler and Bairamov [7].

Example 3.4 (Example 3.2 continued): The mean residual lifetime of a 2-out-of-4 data processing system given that at time $t=10$ at most one display failed could be easily computed through Eq. (3.2). Assume $\left(\lambda_{1}, \lambda_{2}, \lambda_{3}, \lambda_{4}\right)=(0.1,0.3,0.5,0.7)$; it follows that

$$
\mu_{3,2,4}(10)=3.14726 \text {. }
$$

\subsection{Survival Function of $\tilde{X}_{k, l, n, t}$}

The survival function of the residual life of an $(n-k+1)$-out-of- $n$ system given at least $l$ components of the system have failed at time $t \geq 0$ but the system is still working; that is, for $1 \leq l<k \leq n$,

$$
\tilde{X}_{k, l, n, t}=\left[X_{k: n}-t \mid X_{l: n} \leq t, X_{k: n}>t\right]
$$

has a similar mixture representation.

THEOREM 3.5: For $1 \leq l<k \leq n$ and $t, x \geq 0$,

$$
\overline{\tilde{F}}_{k, l, n, t}(x)=P\left(\tilde{X}_{k, l, n, t}>x\right)=\frac{\sum_{i=l}^{k-1} \sum_{C_{i}} \phi_{i}(t) \bar{F}_{k-i, n-i, t}^{\left(C_{i}^{c}\right)}(x)}{\sum_{i=l}^{k-1} \sum_{C_{i}} \phi_{i}(t)} .
$$

Proof: Using arguments similar to these for Theorem 3.1, for $1 \leq l<k \leq n, t, x \geq 0$,

$$
\begin{aligned}
P( & \left.X_{k: n}>t+x, X_{l: n} \leq t, X_{k: n}>t\right) \\
& =P\left(X_{k: n}>t+x, X_{l: n} \leq t\right) \\
& =\sum_{i=l}^{k-1} \sum_{j=0}^{k-i-1} P(\text { exactly } i \text { of } X \text { 's are } \leq t, t<\text { exactly } j \text { of } X \text { 's are } \leq t+x) \\
& =\prod_{i=1}^{n} \bar{F}_{i}(t) \sum_{i=l}^{k-1} \sum_{C_{i}} \phi_{i}(t) \bar{F}_{k-i, n-i, t}^{\left(C_{i}^{c}\right)}(x)
\end{aligned}
$$


and

$$
\begin{aligned}
P\left(X_{l: n} \leq t, X_{k: n}>t\right) & =\sum_{i=l}^{k-1} P(\text { exactly } i \text { of } X \text { 's are } \leq t) \\
& =\sum_{i=l}^{k-1} \sum_{C_{i}} \prod_{s \in C_{i}} F_{s}(t) \prod_{s \in C_{i}^{c}} \bar{F}_{s}(t) \\
& =\prod_{i=1}^{n} \bar{F}_{i}(t) \sum_{i=l}^{k-1} \sum_{C_{i}} \phi_{i}(t) .
\end{aligned}
$$

The required result follows.

Remark: With the help of Theorem 3.5, we get the following representation for the mean residual life function of an $(n-k+1)$-out-of- $n$ system given at least $l$ components of the system have failed at time $t \geq 0$ but the system is still working:

$$
\tilde{\mu}_{k, l, n}(t)=\mathrm{E}\left(\tilde{X}_{k, l, n, t}\right)=\frac{\sum_{i=l}^{k-1} \sum_{C_{i}} \phi_{i}(t) \mu_{k, i, n}^{\left(C_{i}^{c}\right)}(t)}{\sum_{i=l}^{k-1} \sum_{C_{i}} \phi_{i}(t)}, \quad 1 \leq l<k \leq n,
$$

where $\mu_{k, i, n}^{\left(C_{i}^{c}\right)}(t)$ is the same as Eq. (3.2).

Example 3.6: Suppose a plane has four engines and a minimum of two engines are required for the plane work. Hence, this plane is a 2-out-of-4 system. Now, suppose that at time $t$, at least one engine has failed but the system is still working. The residual lifetime of this plane could be modelled as

$$
\tilde{X}_{3,1,4, t}=\left[X_{3: 4}-t \mid X_{1: 4} \leq t, X_{3: 4}>t\right] .
$$

Its survival function is represented as

$$
\bar{F}_{3,1,4, t}(x)=\frac{\sum_{C_{1}} \phi_{1}(t) \bar{F}_{2,3, t}^{\left(C_{1}^{c}\right)}(x)+\sum_{C_{2}} \phi_{2}(t) \bar{F}_{1,2, t}^{\left(C_{2}^{c}\right)}(x)}{\sum_{C_{1}} \phi_{1}(t)+\sum_{C_{2}} \phi_{2}(t)}
$$

Similar to Example 3.2, let us assume $X_{i}$ has an exponential distribution with survival function

$$
\bar{F}_{i}(x)=e^{-\lambda_{i} x}
$$

Note that

$$
\sum_{C_{2}} \phi_{2}(t) \bar{F}_{1,2, t}^{\left(C_{2}^{c}\right)}(x)=\sum_{C_{2}} \prod_{s \in C_{2}}\left(e^{\lambda_{s} t}-1\right) e^{-\sum_{j \in C_{2}^{c}} \lambda_{j} x}
$$




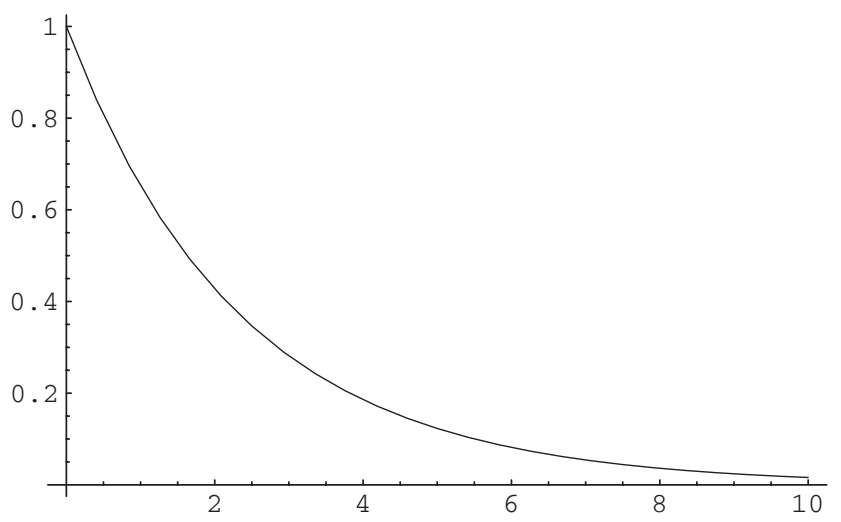

FIGURE 2. The survival function of the residual lifetime of the 2-out-of-4 system given that at least one of the components has failed at time $t=10$.

and

$$
\sum_{C_{2}} \phi_{2}(t)=\sum_{C_{2}} \prod_{s \in C_{2}}\left(e^{\lambda_{s} t}-1\right) .
$$

Now, assume

$$
\left(\lambda_{1}, \lambda_{2}, \lambda_{3}, \lambda_{4}\right)=(0.1,0.3,0.5,0.7) .
$$

The survival function of the system's residual lifetime at time $t=10$ is plotted in Figure 2. The mean residual lifetime of the system given at least one engine has failed at time $t=10$ is

$$
\tilde{\mu}_{3,1,4}(10)=2.37856 \text {. }
$$

\section{STOCHASTIC COMPARISONS}

It is of interest to study the monotone properties of $X_{k, l, n, t}$ and $\tilde{X}_{k, l, n, t}$ with respect $l$. From Corollary 3.2 of Boland, Hollander, Joag-Dev, and Kochar [6] and Theorem 3.2 of Hu and Xie [10], it is known that $X_{k, l, n, t}$ and $\tilde{X}_{k, l, n, t}$ are both stochastically decreasing in $l$, where they considered this problem as the balls and bins experiment. Actually, with the aid of the mixture expressions in Theorems 3.1 and 3.5, one can prove them directly.

The following lemma due to Nanda and Shaked [15] will be used.

LEMMA 4.1: Let $X_{1}, X_{2}, \ldots, X_{m}$ [respectively, $Y_{1}, Y_{2}, \ldots, Y_{n}$ ] be independent (not necessarily i.i.d.) continuous random variables. Then

$$
X_{i} \leq_{\mathrm{st}} Y_{i} \quad \text { for all } i \Rightarrow X_{i: m} \leq_{\mathrm{st}} Y_{j: n}, \quad i \leq j, m-i \geq n-j .
$$


Now, we are ready to present the following direct proof that $X_{k, l, n, t}$ is stochastically decreasing in $l$.

THEOREM 4.2: For $2 \leq l \leq k \leq n$ and $t \geq 0$,

$$
X_{k, l-1, n, t} \geq_{\mathrm{st}} X_{k, l, n, t} \text {. }
$$

PROOF: Note that for $x, t \geq 0, l \geq 2, \bar{F}_{k, l-1, n, t}(x)-\bar{F}_{k, l, n, t}(x)$ has the same sign as

$$
\sum_{i=0}^{l-2} \sum_{C_{i}} \phi_{i}(t) \bar{F}_{k-i, n-i, t}^{\left(C_{i}^{c}\right)}(x) \sum_{i=0}^{l-1} \sum_{C_{i}} \phi_{i}(t)-\sum_{i=0}^{l-1} \sum_{C_{i}} \phi_{i}(t) \bar{F}_{k-i, n-i, t}^{\left(C_{i}^{c}\right)}(x) \sum_{i=0}^{l-2} \sum_{C_{i}} \phi_{i}(t)
$$

that is, for $0 \leq i \leq l-2$,

$$
\begin{aligned}
\sum_{i=0}^{l-2} & \sum_{C_{i}} \phi_{i}(t) \bar{F}_{k-i, n-i, t}^{\left(C_{i}^{c}\right)}(x) \sum_{C_{l-1}} \phi_{l-1}(t)-\sum_{C_{l-1}} \phi_{l-1}(t) \bar{F}_{k-l+1, n-l+1, t}^{\left(C_{l-1}^{c}\right)}(x) \sum_{i=0}^{l-2} \sum_{C_{i}} \phi_{i}(t) \\
= & \sum_{i=0}^{l-2} \sum_{C_{i}} \sum_{C_{l-1}} \prod_{s \in C_{i} \oplus C_{l-1}} \frac{F_{s}(t)}{\bar{F}_{S}(t)} \bar{F}_{k-i, n-i, t}^{\left(C_{i}^{c}\right)}(x) \\
& -\sum_{i=0}^{l-2} \sum_{C_{i}^{\prime}} \sum_{C_{l-1}^{\prime}} \prod_{s \in C_{i}^{\prime} \oplus C_{l-1}^{\prime}} \frac{F_{s}(t)}{\bar{F}_{S}(t)} \bar{F}_{k-l+1, n-l+1, t}^{\left(C_{l-1}^{\prime c}\right)}(x),
\end{aligned}
$$

where $C_{i} \oplus C_{j}$ means the stacking of all elements in two sets $C_{i}$ and $C_{j}$. Note that the first part of the above equation has the same number of terms as the second part, and for each $C_{i}$, there exists $C_{l-1}^{\prime}$ such that $C_{i} \subset C_{l-1}^{\prime}$ and $C_{i}^{\prime} \oplus C_{l-1}^{\prime}=C_{i} \oplus C_{l-1}$. Therefore, the above equation could be written as

$$
\sum_{i=0}^{l-2} \sum_{C_{i} \subset C_{l-1}^{\prime}} \sum_{s \in C_{i} \oplus C_{l-1}} \frac{F_{s}(t)}{\bar{F}_{s}(t)}\left[\bar{F}_{k-i, n-i, t}^{\left(C_{i}^{c}\right)}(x)-\bar{F}_{k-l+1, n-l+1, t}^{\left(C_{l-1}^{\prime c}\right)}(x)\right] .
$$

Since $C_{l-1}^{\prime c} \subset C_{i}^{c}$, according to Lemma 4.1,

$$
\bar{F}_{k-i, n-i, t}^{\left(C_{i}^{c}\right)}(x) \geq \bar{F}_{k-l+1, n-l+1, t}^{\left(C_{l-1}^{\prime c}\right)}(x),
$$

the result follows immediately.

Using a similar argument, one could derive the following result.

THEOREM 4.3: For $t \geq 0$,

$$
\tilde{X}_{k, l-1, n, t} \geq_{\mathrm{st}} \tilde{X}_{k, l, n, t}, \quad 2 \leq l<k \leq n .
$$


Theorem 3.1 of Li and Zhao [14] and Theorem 2.1 of Khaledi and Shaked [13] compares two systems with i.i.d. components from two samples. The following theorem will generalize their results to the case when the components are independent but not identically distributed.

THEOREM 4.4: Let $X_{1}, \ldots, X_{n}, Y_{1}, \ldots, Y_{n}$ be independent continuous random variables. If, for all $1 \leq i, j \leq n, X_{i} \leq_{\mathrm{hr}} Y_{j}$, then for $1 \leq l \leq k \leq n$ and $t \geq 0$,

$$
X_{k, l, n, t} \leq_{\mathrm{st}} Y_{k, l, n, t} .
$$

ProOF: Let $Z$ be a random variable with distribution function $H$ and survival function $\bar{H}$, and it satisfies the following condition: for $1 \leq i, j \leq n$,

$$
X_{i} \leq_{\mathrm{hr}} Z \leq{ }_{\mathrm{hr}} Y_{j} .
$$

Now, let us first prove

$$
X_{k, l, n, t} \leq_{\mathrm{st}} Z_{k, l, n, t} .
$$

Observing Theorem 3.1, it is enough for us to prove

$$
\begin{aligned}
& \sum_{i=0}^{l-1}\left(\begin{array}{c}
n \\
i
\end{array}\right) \phi_{i}^{Z}(t) \bar{H}_{k-i, n-i, t}(x) \sum_{i=0}^{l-1} \sum_{C_{i}} \phi_{i}^{X}(t)-\sum_{i=0}^{l-1} \sum_{C_{i}} \phi_{i}^{X}(t) \bar{F}_{k-i, n-i, t}^{\left(C_{i}^{c}\right)}(x) \\
& \quad \times \sum_{i=0}^{l-1}\left(\begin{array}{c}
n \\
i
\end{array}\right) \phi_{i}^{Z}(t) \geq 0,
\end{aligned}
$$

where

$$
\phi_{i}^{Z}(t)=\left[\frac{H(t)}{\bar{H}(t)}\right]^{i}, \quad \phi_{i}^{X}(t)=\prod_{s \in C_{i}} \frac{F_{s}(t)}{\bar{F}_{s}(t)} .
$$

Note that the above expression could be represented as, for $t \geq 0$ and $x \geq 0$,

$$
\begin{aligned}
& \sum_{i=0}^{l-1} \sum_{j=0}^{l-1} \sum_{C_{j}} \phi_{j}^{X}(t)\left(\begin{array}{c}
n \\
i
\end{array}\right) \phi_{i}^{Z}(t) \bar{H}_{k-i, n-i, t}(x)-\sum_{i=0}^{l-1} \sum_{j=0}^{l-1} \sum_{C_{j}}\left(\begin{array}{c}
n \\
i
\end{array}\right) \phi_{i}^{Z}(t) \phi_{j}^{X}(t) \bar{F}_{k-j, n-j, t}^{\left(C_{j}^{c}\right)}(x) \\
& =\sum_{i=0}^{l-1} \sum_{j=0}^{l-1} \sum_{C_{j}}\left(\begin{array}{c}
n \\
i
\end{array}\right) \phi_{i}^{Z}(t) \phi_{j}^{X}(t)\left[\bar{H}_{k-i, n-i, t}(x)-\bar{F}_{k-j, n-j, t}^{\left(C_{j}^{c}\right)}(x)\right] \\
& =\Delta_{1}+\Delta_{2},
\end{aligned}
$$

where

$$
\Delta_{1}=\sum_{i=0}^{l-1} \sum_{j=0}^{l-1} \sum_{C_{j}}\left(\begin{array}{l}
n \\
i
\end{array}\right) \phi_{j}^{X}(t) \phi_{i}^{Z}(t)\left[\bar{H}_{k-i, n-i, t}(x)-\bar{H}_{k-j, n-j, t}(x)\right]
$$


and

$$
\Delta_{2}=\sum_{i=0}^{l-1} \sum_{j=0}^{l-1} \sum_{C_{j}}\left(\begin{array}{c}
n \\
i
\end{array}\right) \phi_{j}^{X}(t) \phi_{i}^{Z}(t)\left[\bar{H}_{k-j, n-j, t}(x)-\bar{F}_{k-j, n-j, t}^{\left(C_{j}^{c}\right)}(x)\right] .
$$

According to Eq. (2.3) and Lemma 4.1, it is seen that, for any $x \geq 0, j=1, \ldots, l$,

$$
\bar{F}_{k-j, n-j, t}^{\left(C_{j}^{c}\right)}(x) \leq \bar{H}_{k-j, n-j, t}(x) \text {; }
$$

hence, $\Delta_{2} \geq 0$.

So, it is sufficient to show $\Delta_{1} \geq 0$. Observe

$$
\begin{aligned}
\Delta_{1}= & \sum_{i=0}^{l-1} \sum_{j=i}^{l-1} \sum_{C_{j}}\left(\begin{array}{l}
n \\
i
\end{array}\right) \phi_{j}^{X}(t) \phi_{i}^{Z}(t)\left[\bar{H}_{k-i, n-i, t}(x)-\bar{H}_{k-j, n-j, t}(x)\right] \\
& +\sum_{i=0}^{l-1} \sum_{j=0}^{i} \sum_{C_{j}}\left(\begin{array}{c}
n \\
i
\end{array}\right) \phi_{j}^{X}(t) \phi_{i}^{Z}(t)\left[\bar{H}_{k-i, n-i, t}(x)-\bar{H}_{k-j, n-j, t}(x)\right] \\
= & \sum_{i=0}^{l-1} \sum_{j=i}^{l-1} \sum_{C_{j}}\left(\begin{array}{l}
n \\
i
\end{array}\right) \phi_{j}^{X}(t) \phi_{i}^{Z}(t)\left[\bar{H}_{k-i, n-i, t}(x)-\bar{H}_{k-j, n-j, t}(x)\right] \\
& +\sum_{i=0}^{l-1} \sum_{j=i}^{l-1} \sum_{C_{i}}\left(\begin{array}{l}
n \\
j
\end{array}\right) \phi_{i}^{X}(t) \phi_{j}^{Z}(t)\left[\bar{H}_{k-j, n-j, t}(x)-\bar{H}_{k-i, n-i, t}(x)\right] \\
= & \sum_{i=0}^{l-1} \sum_{j=i}^{l-1}\left[\bar{H}_{k-i, n-i, t}(x)-\bar{H}_{k-j, n-j, t}(x)\right] \\
& \times\left[\sum_{C_{j}}\left(\begin{array}{c}
n \\
i
\end{array}\right) \phi_{j}^{X}(t) \phi_{i}^{Z}(t)-\sum_{C_{i}}\left(\begin{array}{c}
n \\
j
\end{array}\right) \phi_{i}^{X}(t) \phi_{j}^{Z}(t)\right] .
\end{aligned}
$$

From Lemma 4.1, it follows that, for $i \leq j, x \geq 0$,

$$
\bar{H}_{k-i, n-i, t}(x) \geq \bar{H}_{k-j, n-j, t}(x) .
$$

Note that, for $i \leq j$,

$$
\begin{aligned}
& \sum_{C_{j}}\left(\begin{array}{c}
n \\
i
\end{array}\right) \phi_{j}^{X}(t) \phi_{i}^{Z}(t)-\sum_{C_{i}}\left(\begin{array}{l}
n \\
j
\end{array}\right) \phi_{i}^{X}(t) \phi_{j}^{Z}(t) \\
& =\sum_{C_{j}}\left(\begin{array}{c}
n \\
i
\end{array}\right) \prod_{s \in C_{j}} \frac{F_{s}(t)}{\bar{F}_{s}(t)}\left[\frac{H(t)}{\bar{H}(t)}\right]^{i}-\sum_{C_{i}}\left(\begin{array}{l}
n \\
j
\end{array}\right) \prod_{s \in C_{i}} \frac{F_{s}(t)}{\bar{F}_{s}(t)}\left[\frac{H(t)}{\bar{H}(t)}\right]^{j} \\
& =\sum_{C_{j}} \prod_{s \in C_{j}} \frac{F_{s}(t)}{\bar{F}_{s}(t)} \sum_{C_{i}}\left[\frac{H(t)}{\overline{\bar{H}}(t)}\right]^{i}-\sum_{C_{i}} \prod_{s \in C_{i}} \frac{F_{S}(t)}{\bar{F}_{s}(t)} \sum_{C_{j}}\left[\frac{H(t)}{\bar{H}(t)}\right]^{j}
\end{aligned}
$$


Since the first part has the same number of terms as the second part, for each $C_{i}$ we could find $C_{j}$ such that $C_{i} \subset C_{j}$. Hence, the above equation could be written as

$$
\sum_{C_{i} \subset C_{j}} \prod_{s \in C_{i}} \frac{F_{s}(t)}{\bar{F}_{s}(t)}\left[\frac{H(t)}{\bar{H}(t)}\right]^{i}\left[\prod_{s \in C_{j}-C_{i}} \frac{F_{s}(t)}{\bar{F}_{s}(t)}-\left(\frac{H(t)}{\bar{H}(t)}\right)^{j-i}\right] \geq 0,
$$

where the inequality follows from Eq. (2.2).

Hence, relation (4.1) is true. Using a similar argument, it can also be proved that

$$
Z_{k, l, n, t} \leq_{\mathrm{st}} Y_{k, l, n, t} .
$$

Combing the two results, the required result follows.

One can derive the following result by a similar argument.

THeOREM 4.5: Let $X_{1}, \ldots, X_{n}, Y_{1}, \ldots, Y_{n}$ be independent continuous random variables. If, for all $1 \leq i, j \leq n, X_{i} \leq_{\mathrm{hr}} Y_{j}$, then for $1 \leq l \leq k \leq n$ and $t \geq 0$,

$$
\tilde{X}_{k, l, n, t} \leq_{\mathrm{st}} \tilde{Y}_{k, l, n, t}
$$

\section{APPLICATIONS IN PHR MODELS}

The proportional hazards model (PHR) has been widely used in reliability theory, survival analysis and engineering, and so forth, due to its simplicity and flexibility. Let $X_{1}, X_{2}, \ldots, X_{n}$ be independent random variables. They are said to follow the PHR model if for $i=1,2, \ldots, n$, the survival function of $X_{i}$ can be expressed as

$$
\bar{F}_{i}(x)=[\bar{F}(x)]^{\lambda_{i}} \quad \text { for } \lambda_{i}>0,
$$

where $\bar{F}(x)$ is the survival function of some random variable $X$. If $r(t)$ denotes the hazard rate corresponding to the baseline distribution $F$, then the hazard rate of $X_{i}$ is $\lambda_{i} r(t), i=1,2, \ldots, n$. It includes many well-known models such as exponential, Weibull, Rayleigh, and Pareto.

Equation (3.1) enables us to derive a dynamic bound for the hazard rate function of the residual lifetime of a parallel system given that all of the components are working at time $t$ :

$$
X_{n, 1, n, t}=\left[X_{n: n}-t \mid X_{1: n}>t\right],
$$

which is of particular interest in practice.

Proposition 5.1: Let $X_{1}, \ldots, X_{n}$ be independent random variables with $X_{i}$ having survival function $\bar{F}^{\lambda_{i}}, i=1, \ldots, n$, and let $Y_{1}, \ldots, Y_{n}$ be another random sample with the common survival function $\bar{F}^{\lambda}$. Then

$$
\lambda \geq \tilde{\lambda}=\left(\prod_{i=1}^{n} \lambda_{i}\right)^{1 / n} \Longrightarrow X_{n, 1, n, t} \geq_{\mathrm{hr}} Y_{n, 1, n, t} .
$$


ProOF: According to Eq. (3.1),

$$
X_{n, 1, n, t} \stackrel{\text { st }}{=} X_{n, n, t}
$$

the largest order statistic from $\left(X_{i}\right)_{t}$ for $i=1, \ldots, n$. Note that for $i=1, \ldots, n$,

$$
P\left(\left(X_{i}\right)_{t}>x\right)=\left[\frac{\bar{F}(t+x)}{\bar{F}(t)}\right]^{\lambda_{i}}
$$

belongs to the PHR model. From Theorem 4.6 of Khaledi and Kochar [12], the required result follows.

Example 5.2: Let $X_{1}, \ldots, X_{n}$ be independent Weibull random variables with $X_{i}$ having survival function, for $i=1, \ldots, n$,

$$
\bar{F}_{i}(x)=e^{-\lambda_{i} x^{2}}, \quad \lambda_{i}>0, x>0 .
$$

The hazard rate of $X_{n, n, t}$ is

$$
r_{n, n, t}(x)=\frac{\prod_{i=1}^{n}\left(1-e^{-\lambda_{i}\left(x^{2}+2 x t\right)}\right)}{1-\prod_{i=1}^{n}\left(1-e^{-\lambda_{i}\left(x^{2}+2 x t\right)}\right)} \sum_{i=1}^{n} \frac{2 \lambda_{i}(x+t) e^{-\lambda_{i}\left(x^{2}+2 x t\right)}}{1-e^{-\lambda_{i}\left(x^{2}+2 x t\right)}} .
$$

According to Proposition 5.1, it holds that

$$
r_{n, n, t}(x) \leq \frac{2 n \tilde{\lambda}(x+t) e^{-\tilde{\lambda}\left(x^{2}+2 x t\right)}\left(1-e^{-\tilde{\lambda}\left(x^{2}+2 x t\right)}\right)^{n-1}}{1-\left(1-e^{-\tilde{\lambda}\left(x^{2}+2 x t\right)}\right)^{n}}
$$

Now, assume $n=3$ and

$$
\left(\lambda_{1}, \lambda_{2}, \lambda_{3}\right)=(0.1,0.2,0.3)
$$

In Figure 3, we plot the hazard rate functions of residual lifetimes of the parallel system given that all of the components are working at time $t$ when (1) the parameters of distributions are $\left(\lambda_{1}, \lambda_{2}, \lambda_{3}\right)$, (2) when the common parameters are the arithmetic mean $(0.2,0.2,0.2)$, and (3) when the common parameter is the geometric mean $(0.182,0.182,0.182)$ of $\left(\lambda_{1}, \lambda_{2}, \lambda_{3}\right)$ at time $t=10$. It can be seen that the bound in case of the geometric mean is better than that for the arithmetic mean.

Due to the complicated expression of the survival function of the residual lifetime of a $k$-out-of- $n$ system with independent but nonidentical components, it will be of interest to provide upper and lower bounds for the survival function of the system's residual lifetime. According to Theorems 4.4 and 4.5, we have the following results. 


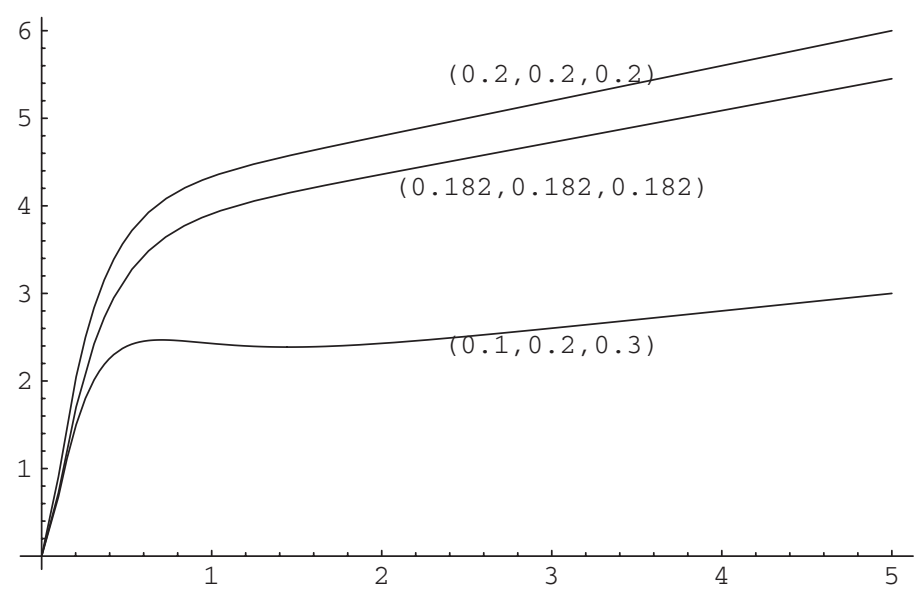

FigURE 3. The hazard rate functions of the residual lifetimes of the 1-out-of-3 system given that all of the components are working at time $t=10$ with different parameters.

Proposition 5.3: Let $X_{1}, \ldots, X_{n}$ be independent random variables with $X_{i}$ having survival function $\bar{F}^{\lambda_{i}}, i=1, \ldots, n$. Then

$$
\bar{H}_{k, l, n, t}(x) \leq \bar{F}_{k, l, n, t}(x) \leq \bar{G}_{k, l, n, t}(x)
$$

where $\bar{H}_{k, l, n, t}(x)$ is the survival function of $X_{k, l, n, t}$ from i.i.d. random variables $X_{1}, \ldots, X_{n}$ with the common parameter $\lambda=\max \left\{\lambda_{1}, \ldots, \lambda_{n}\right\}$ and $\bar{G}_{k, l, n, t}(x)$ is the survival function of $X_{k, l, n, t}$ with the common parameter $\lambda=\min \left\{\lambda_{1}, \ldots, \lambda_{n}\right\}$.

Proposition 5.4: Let $X_{1}, \ldots, X_{n}$ be independent random variables with $X_{i}$ having survival function $\bar{F}^{\lambda_{i}}, i=1, \ldots, n$. Then

$$
\overline{\tilde{H}}_{k, l, n, t}(x) \leq \overline{\tilde{F}}_{k, l, n, t}(x) \leq \overline{\tilde{G}}_{k, l, n, t}(x)
$$

where $\overline{\tilde{H}}_{k, l, n, t}(x)$ is the survival function of $\tilde{X}_{k, l, n, t}$ from i.i.d. random variables $X_{1}, \ldots, X_{n}$ with the common parameter $\lambda=\max \left\{\lambda_{1}, \ldots, \lambda_{n}\right\}$, and $\overline{\tilde{G}}_{k, l, n, t}(x)$ is the survival function of $\tilde{X}_{k, l, n, t}$ with the common parameter $\lambda=\min \left\{\lambda_{1}, \ldots, \lambda_{n}\right\}$.

Example 5.5 (Example 3.2 continued): Assume

$$
\left(\lambda_{1}, \lambda_{2}, \lambda_{3}, \lambda_{4}\right)=(0.2,0.25,0.3,0.35) .
$$

One can use $\lambda_{1}=0.2$ and $\lambda_{4}=0.35$ to provide upper and lower bounds for the survival function of the residual lifetime of the 2-out-of-4 system given at most one display failed at time $t=10$ (see Fig. 4). 


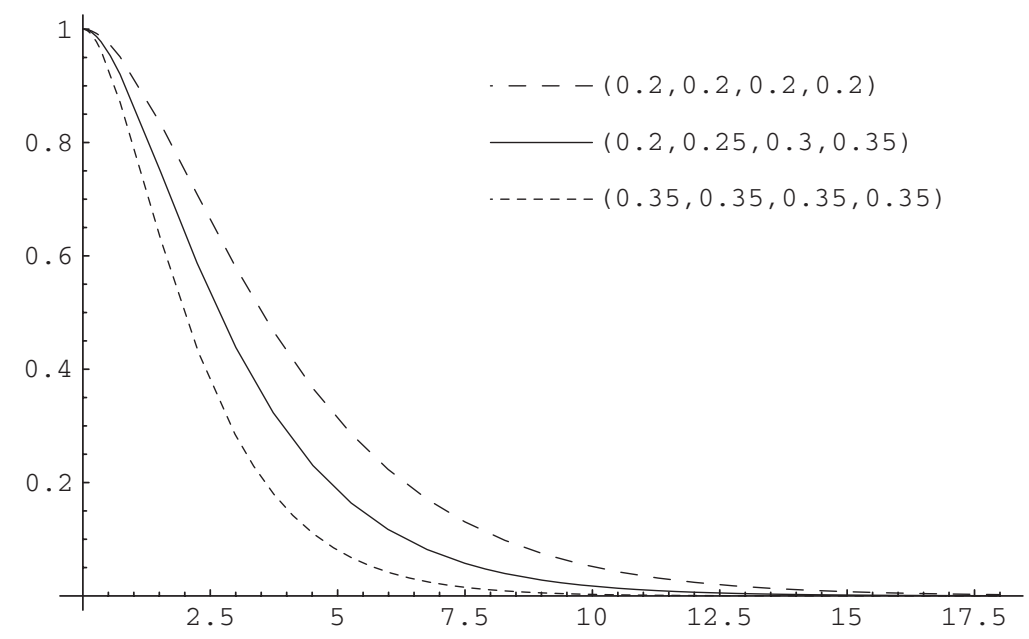

FIGURE 4. Bounds for the survival function of the residual lifetime of the 2-out-of-4 system given at most one display failed at time $t=10$.

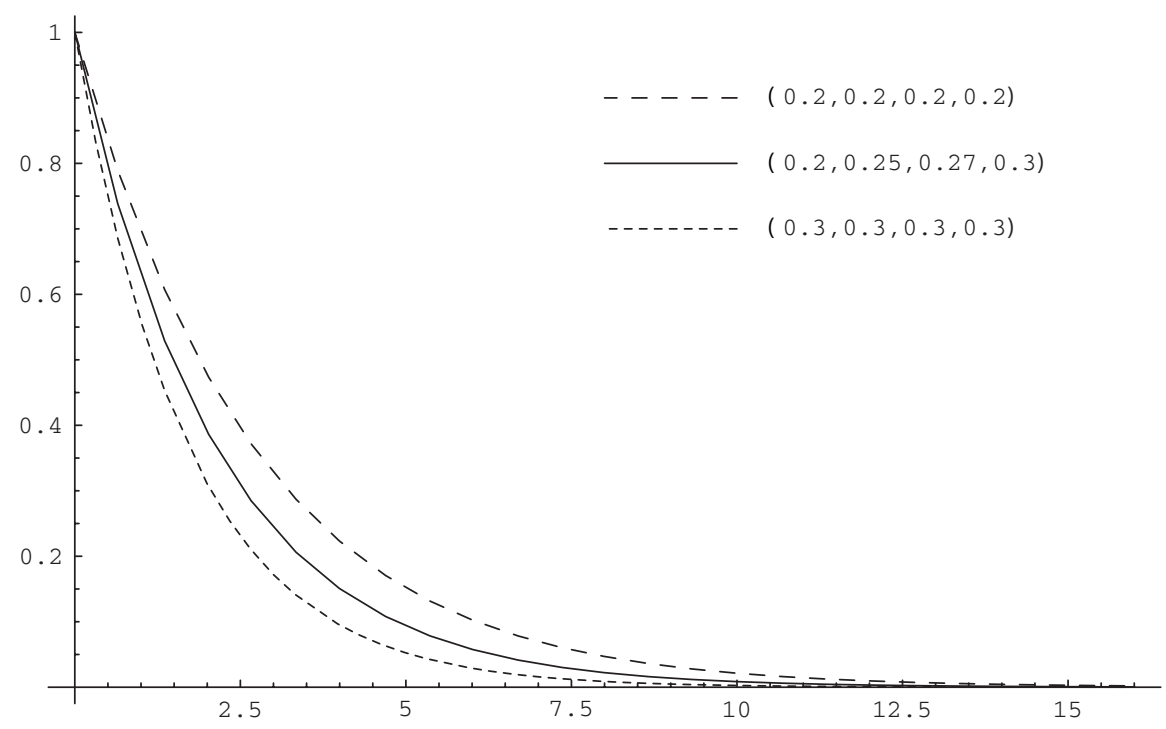

Figure 5. Bounds for the survival function of the residual lifetime of the 2-out-of-4 system given that at least one engine failed but the system is still working at time $t=10$.

Example 5.6 (Example 3.6 continued): Assume

$$
\left(\lambda_{1}, \lambda_{2}, \lambda_{3}, \lambda_{4}\right)=(0.2,0.25,0.27,0.3) .
$$


One can use $\lambda_{1}=0.2$ and $\lambda_{4}=0.3$ to provide upper and lower bounds for the survival function of the residual lifetime of the 2-out-of- 4 system given that at least one engine has failed but the system is still working at time $t=10$ (see Fig. 5).

\section{Acknowledgments}

The authors are grateful to the anonymous referee for his/her insightful comments and suggestions that have led to an improved version of the article.

\section{References}

1. Asadi, M. \& Bairamov, I. (2005). A note on the mean residual life function of a parallel system. Communications in Statistics: Theory and Methods 34: 475-485.

2. Asadi, M. \& Bairamov, I. (2006). The mean residual life function of a $k$-out-of- $n$ structure at the system level. IEEE Transactions on Reliability 55: 314-318.

3. Bairamov, I., Ahsanullah, M. \& Akhundov, I. (2002). A residual life function of a system having parallel or series structures. Journal of Statistical Theory and Application 1: 119-132.

4. Balakrishnan, N. (2007). Permanents, order statistics, outliers, and robustness. Revista Matemática Complutense 20: 7-107.

5. Bapat, R.B. \& Kochar, S.C. (1994). On likelihood-ratio ordering of order statistics. Linear Algebra and Its Applications 199: 281-291.

6. Boland, P.J., Hollander, M., Joag-Dev, K. \& Kochar, S. (1996). Biviariate dependence properties of order statistics. Journal of Multivariate Analysis 56: 75-89.

7. Gurler, S. \& Bairamov, I. (2009). Parallel and $k$-out-of- $n$ : G systems with nonidentical components and their mean residual life functions. Applied Mathematical Modelling 33: 1116-1125.

8. Hu, T., Jin, W. \& Khaledi, B.-E. (2007). Ordering conditional distributions of generalized order statistics. Probability in the Engineering and Informational Sciences 21: 401-417.

9. Hu, T., Wang, F. \& Zhu, Z. (2006). Stochastic comparisons and dependence of spacings from two samples of exponential random variables. Communications in Statistics: Theory and Methods 35: 979-988.

10. Hu, T. \& Xie, C. (2006). Negative dependence in the balls and bins experiment with applications to order statistics. Journal of Multivariate Analysis 97: 1342-1354.

11. Hu, T., Zhu, Z. \& Wei, Y. (2001). Likelihood ratio and mean residual life orders for order statistics of heterogeneous random variables. Probability in the Engineering and Informational Sciences 15: $259-272$.

12. Khaledi, B.-E. \& Kochar, S.C. (2002). Stochastic orderings among order statistics and sample spacings. In J.C. Misra (ed.), Uncertainty and Optimality-probability, statistics and operations research. Singapore: World Scientific Publications, pp. 167-203.

13. Khaledi, B.-E. \& Shaked, M. (2007). Ordering conditional lifetimes of coherent systems. Journal of Statistical Planning and Inference 137: 1173-1184.

14. Li, X. \& Zhao, P. (2006). Some aging properties of the residual life of $k$-out-of- $n$ systems. IEEE Transactions on Reliability 55: 535-541.

15. Nanda, A.K. \& Shaked, M. (2001). The hazard rate and the reversed hazard rate orders, with applications to order statistics. Annals of the Institute of Statistical Mathematics 53: 853-864.

16. Navarro, J., Balakrishnan, N. \& Samaniego, F.J. (2008). Mixture representations of residual lifetimes of used systems. Journal of Applied Probability 45: 1097-1112.

17. Sadegh, M.K. (2008). Mean past and mean residual life functions of a parallel system with nonidentical components. Communications in Statistics Theory and Methods 37: 1134-1145.

18. Shaked, M. \& Shanthikumar, J.G. (2007). Stochastic orders and their applications. New York: Springer.

19. Zhao, P., Li, X. \& Balakrishnan, N. (2008). Conditional ordering of $k$-out-of- $n$ systems with independent but nonidentical components. Journal of Applied Probability 45: 1113-1125. 